\title{
Plant DNA Recombinases: A Long Way to Go
}

\author{
Rajani Kant Chittela and Jayashree K. Sainis \\ Plant Biochemistry Section, Molecular Biology Division, Bhabha Atomic Research Center, \\ Trombay, Mumbai 400 085, India
}

Correspondence should be addressed to Jayashree K. Sainis, jksainis@barc.gov.in

Received 8 July 2009; Accepted 8 September 2009

Academic Editor: Aidan Doherty

Copyright ( $) 2010$ R. K. Chittela and J. K. Sainis. This is an open access article distributed under the Creative Commons Attribution License, which permits unrestricted use, distribution, and reproduction in any medium, provided the original work is properly cited.

\begin{abstract}
DNA homologous recombination is fundamental process by which two homologous DNA molecules exchange the genetic information for the generation of genetic diversity and maintain the genomic integrity. DNA recombinases, a special group of proteins bind to single stranded DNA (ssDNA) nonspecifically and search the double stranded DNA (dsDNA) molecule for a stretch of DNA that is homologous with the bound ssDNA. Recombinase A (RecA) has been well characterized at genetic, biochemical, as well as structural level from prokaryotes. Two homologues of RecA called Rad51 and Dmc1 have been detected in yeast and higher eukaryotes and are known to mediate the homologous recombination in eukaryotes. The biochemistry and mechanism of action of recombinase is important in understanding the process of homologous recombination. Even though considerable progress has been made in yeast and human recombinases, understanding of the plant recombination and recombinases is at nascent stage. Since crop plants are subjected to different breeding techniques, it is important to know the homologous recombination process. This paper focuses on the properties of eukaryotes recombinases and recent developments in the field of plant recombinases Dmc1 and Rad51.
\end{abstract}

\section{Homologous Recombination}

The homologous recombination (HR) is a fundamental, vital and conservative process in all living organisms. This process is important for generation of genetic diversity, maintaining the genomic integrity as well as repairing the double strand breaks which are generated by the action of ionizing radiation, exposure to genotoxic chemicals, errors in replication, and during cell development (Figure 1) [1,2].

The importance of homologous recombination in prokaryotes and eukaryotes was realized a long time ago. Mutants sensitive to DNA damaging agents like UV and ionizing radiation showed deficiency in recombination [35]. The complex nature of the process and crosstalk between homologous recombination and other pathways like transcription, repair, and replication posed a great challenge to the biologists [6].

The genetics and biochemistry of HR process was best understood in simple prokaryote E. coli, which shows high frequency of homologous recombination. Various recombination deficient mutants were generated and investigated for their phenotypes showing that more than 20 genes are important in this process [7]. Homologous recombination in E. coli occurs mainly by the RecBCD pathway. The gene products recA, recB, recC, recD, recF, recG, recJ, recN, recO, recR, ruvA, ruvB, ruvC, and ssb are known to play role in different steps of recombination such as initiation of DNA-DNA interaction, homology search, strand exchange, branch migration, and Holliday junction resolution. Among all these, the most important step is homology search, which is carried out by a special group of proteins called recombinase A (RecA).

\section{Recombinase A (RecA) from $E$. coli}

E. coli recA plays an important role in induced stable DNA replication, constitutive stable DNA replication, and chromosome partitioning [7]. Almost 20 years after the discovery of the recA gene in E. coli, RecA protein was purified. It is a $37.8 \mathrm{kDa}$ protein with 352 amino acids and has different biochemical activities [7]. It binds to single and double stranded DNA to form right handed helical 


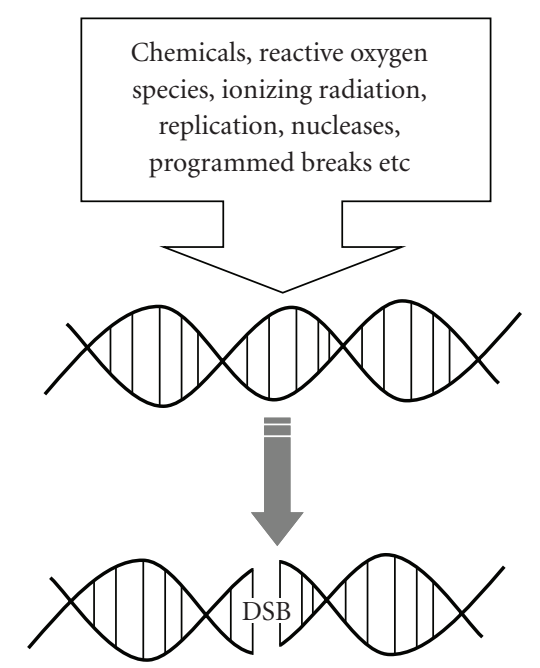

Figure 1: DNA damage caused by different genotoxic agents. The figure shows the formation of double strand breaks (DSBs) in dsDNA when exposed to DNA damaging agents like genotoxic chemicals, ionizing radiation as well as errors in the replication, programmed and unprogrammed action of nucleases.

filaments and has ssDNA dependent ATPase activity. E. coli RecA mediates renaturation and strand exchange functions, which are the two main activities required for the process of homologous recombination. RecA protein after binding (one monomer to three nucleotides) to single stranded DNA, stretches it 1.5 times to its original length. This stretching process exposes the DNA bases, which are essential for homologous sequence search in renaturation and strand exchange reaction. Renaturation of complementary single strands into duplex DNA molecules was the first activity demonstrated for RecA protein [8]. This reaction serves as an initial step. Renaturation reaction was optimal at low concentrations of RecA and in the presence of ATP [9]. The functional significance of the renaturation reaction is to increase the effective DNA concentration by DNA aggregation process $[10,11]$. Once the effective local concentration is achieved, RecA promotes the strand exchange reaction between homologous sequence partners. Studies on molecular mechanism of action of E. coli RecA have shown that RecA protein, in presence of ATP, binds to ssDNA to form ternary complex called presynaptic filament $[10,12]$. Single stranded DNA binding protein was shown to accelerate the filament formation by removing the secondary structures in the DNA. This presynaptic filament, where one protomer binds to three nucleotides, searches the homologous sequence in the duplex partner and mediates a three stranded pairing called synaptic filament. The synaptic filament forms homologous joint molecules containing displacement loop structures called Dloops. At this stage, the newly formed double stranded DNA has the old parental strand and a new incoming strand. It is therefore called heteroduplex. These heteroduplexes further extended to mediate complete strand exchange [7].

RecA also binds and hydrolyzes ATP to mediate the renaturation and strand exchange. It was assumed that the energy released by ATP hydrolysis drives the strand exchange reaction. But the renaturation as well as the strand exchange processes are isoenergitic reactions where energy released by duplex formation drives the opening of the duplex at the other site. The overall energy is conserved in this process. The studies on RecA protein in the presence of nonhydrolysable ATP analogues like AMP-PNP and ATP$\gamma$-S showed that extensive heteroduplex formation occurs without much of ATP hydrolysis [13-15]. Hence it is believed that the chemical energy derived from ATP hydrolysis is not required for homologous pairing and strand exchange. Differential binding affinities of RecA towards the ADP and ATP make the protein cycling for the reactions, where RecA-ATP complex has more affinity towards DNA than RecA-ADP [13, 14]. Additionally, ATP dependent motor function was assigned to RecA protein. Two models have been proposed for motor type function of RecA protein [16]. In the first model free energy released upon ATP hydrolysis is hypothesized to help in redistribution of RecA monomers on DNA substrates during the strand exchange reactions. In the second model, ATP hydrolysis is envisaged to help in rotation of the DNA substrates.

Thus prokaryotic RecA was shown to have the required biochemical properties for mediating the homology search and strand exchange in homologous recombination. Since homologous recombination is a conserved process from simple prokaryotes to higher eukaryotes, the information on the biochemistry and genetics of RecA protein led to the foundation for searching the eukaryotic homolgues of recombinases. With yeast being a simple eukaryotic system, many studies were done in this system to unravel function of recombinase at genetic and biochemical level.

\section{Recombinases in Yeast}

Homologous recombination in yeast occurs during meiosis, mating type switching, and DSB repair. Homologous recombination in yeast plays a major role in DNA repair as compared to the NHEJ. The yeast cells compromised for NHEJ do not show any increase in sensitivity to DNA damaging agents; whereas cells with nonfunctional HR and NHEJ show increased sensitivity. Based on the genetic and biochemical studies, several genes have been discovered belonging to RAD52 epistasis group, which include RAD50, RAD51, RAD52, RAD54, RAD55, RAD57, RAD59, RDH54/TID, MRE11, and XRS2. Figure 2 shows various steps in homologous recombination and the proteins participating in this process in eukaryotes. Based on the sequence homology, two types of RecA homologues have been reported in yeast, namely, ScRad51 and ScDmc1. Rad51 expresses during mitosis and meiosis where as Dmc1 expresses only during meiosis [17]. However, DMC1 is not considered in RAD52 epistasis group of genes though it is important for meiotic recombination [2].

RAD51 mutants in yeast were isolated during the screening for the sensitivity to ionizing radiation and showed reduced mitotic and meiotic recombination. These mutants also showed sensitivity to genotoxic chemicals 
like adriamycin, bleomycine, methyl methane sulphonate, etc [18]. RAD51 mutants of yeast have poor sporulation efficiency, accumulation of meiotic DSBs, low spore viability; however null mutants of RAD51 are viable $[5,19]$. Sequence comparison also showed that yeast $\operatorname{Rad} 51$ protein is a functional and structural homologue of E. coli RecA. Yeast Rad51 protein shares 29\% identity and 54\% homology with RecA protein. ATPase activity of yeast Rad51 is stimulated by ssDNA. However, when compared with RecA, yeast Rad51 exhibits limited strand exchange activity [20].

The other recombinase Disrupted Meiotic cDNA1 (DMC1) was detected from yeast during the studies on isolation of cDNA expressed during the meiosis. DMC1 disruption resulted in defects in reciprocal recombination, inability to form synaptonemal complex, accumulation of DSBs, and abnormal chromosome synapses [21]. It shared $28 \%$ identity and $41 \%$ similarity with RecA. Biochemical investigations showed that Dmcl from yeast also binds to single and double strands to form helical filaments, promotes renaturation of complementary single strands into duplex molecules, and shows D-loop formation and strand exchange activities [22]. AFM studies have also shown that ScDmc1 forms $90 \%$ octameric ring like structure as well as $10 \%$ helical filaments upon binding to ssDNA. The helical forms are hypothesized to represent the active forms responsible for recombination reactions [23].

\section{Recombinases in Mammals}

Homologous recombination has been shown to be important for DSB repair in higher eukaryotes [2]. Based on the information available from $E$. coli and yeast, two orthologues of recombinases, namely, DMC1 and RAD51 were identified in mammalian systems. Human DMC1 was isolated and shown to be a RecA homologue [24]. Male and female mice with mutations in DMC1 were sterile, where gametogenesis was arrested in prophase and meiotic chromosomes failed to synapse $[25,26]$.

Using the yeast $R A D 51$ as a probe, human and mice $R A D 51$ genes were isolated and cloned [27-29]. It was shown that a mutation in $R A D 51$ led to an early embryonic lethality in mice, which was suppressed by corresponding mutation in p53 [30]. Formation of the Rad51 nuclear foci in PHAstimulated lymphocytes was thought to be involved in DNA recombination or DNA repair in S phase [31]. It was also shown that Rad51 functions in late $S$ and G2 phases of cell cycle in peripheral blood lymphocytes [32]. RAD51 was shown to be responsible for the higher recombination activities in cancer cell line [33].

Although mammalian RAD51 genes were reported from human and mouse only human Rad51 protein was subjected to biochemical analysis. Like RecA, hRad51 protein also binds to ssDNA and dsDNA to form helical filaments and mediates strand exchange reaction. RecA was shown to mediate branch migration in $5^{\prime}$ to $3^{\prime}$ direction whereas hRad51 protein catalyzed branch migration in both the directions [34, 35]. Human Rad51 showed homologous pairing and strand exchange activities in ATP and homology

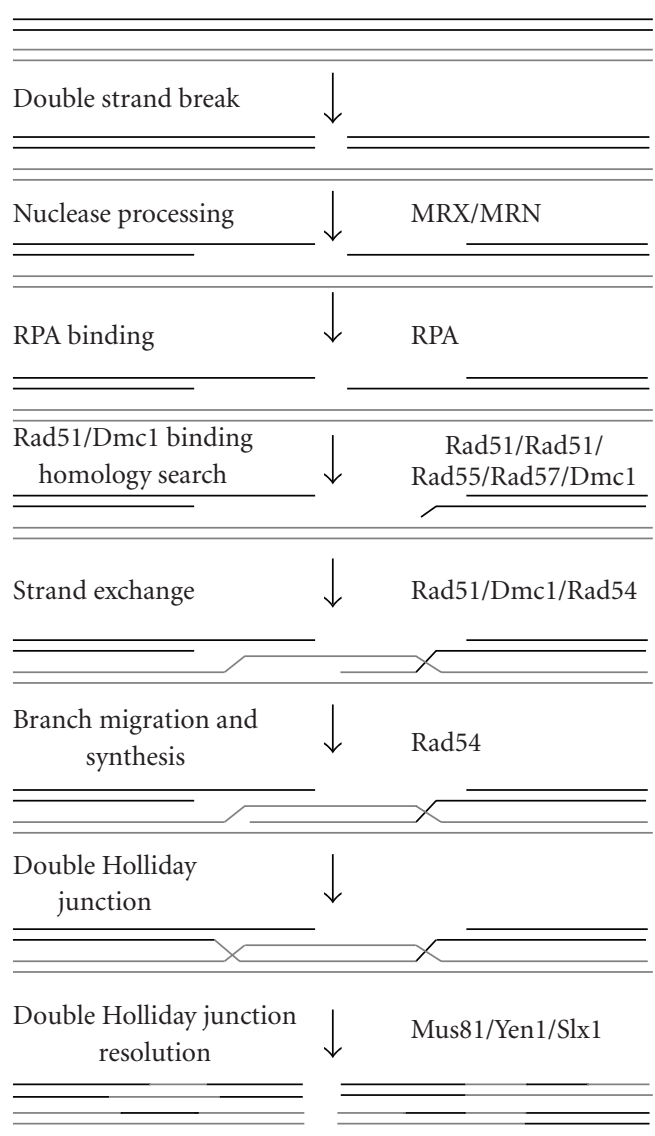

Figure 2: Biochemical steps and corresponding proteins involved in homologous recombination in eukaryotes. Left hand side of the figure shows the different steps involved in the process and the corresponding proteins responsible for the process are shown on the right. The double strand break introduced in one of the two homologous DNA molecules is subjected to nucleolytic processing by MRX/MRN complex to expose $3^{\prime}$ ends. With the help of RPA, Rad52, Rad54, Rad55, Rad57, and Rad51/Dmc1, the ssDNA locates the complementary region on homologous DNA molecule resulting in strand invasion followed by strand exchange. Rad54 helps in branch migration. The $3^{\prime}$ ends are used as primers for new DNA synthesis at gap site. Resulted Holliday junction is resolved to generate repaired molecules.

dependent manner. These activities were found to be stimulated in the presence of RPA [36]. Human Rad51 also required ATP for DNA binding and for mediating the homologous pairing $[37,38]$. The hRad51 was shown to bind preferentially to the single stranded tailed duplexes with higher affinity over that of single and double stranded oligonucleotides [39]. Using Fluorescence Resonance Energy Transfer, hDmcl was shown to catalyze the strand exchange and strand assimilation in homology dependent manner [40]. In co-operation with a heterotrimeric protein, replication protein A (RPA), hDMC1 performed strand exchange reaction up to 5.4 kilo base pairs in vitro [41, 42]. Passy et al. [43] and Masson et al. [44] have demonstrated that human Dmcl forms octameric ring like structures on ssDNA. Presence of ATP was found to result in the formation 
of helical filaments on ssDNA, whereas in the absence of ATP there is more preponderance of octameric rings [42].

In addition to this, five $R A D 51$ paralogs, $R A D 51 L 1 / B$, RAD51L2/C, RAD51L3/D, XRCC2, and XRCC3 have been identified in mammals [25, 45-50]. These paralogs showed around 25-30\% homology with RAD51 [51]. RAD51L1, RAD51L3, and XRCC2 targeted mice showed embryonic lethality [52-54]. In humans, these paralogs facilitated the Rad51 mediated homologous recombination $[17,55,56]$.

\section{Structural Studies on DNA Recombinases}

Three-dimensional structures provide the framework for understanding the biochemical and genetic data. Threedimensional crystal structures of RecA in the presence and absence of ADP have been solved by Story et al. in 1992 [57, 58]. It was observed that RecA in the presence of ADP forms less affinity complex and in the absence of ADP or presence of ATP forms high affinity complex with ssDNA. Based on the three-dimensional structure it was suggested that RecA protein consists of a central domain with Walker motifs responsible for ATP binding and its hydrolysis. This central domain may also interact with DNA. In addition to this central domain, loop 1 and loop 2 were proposed to be involved in DNA binding. These observations were supported by the observation that mutations within these regions abolished DNA binding and ATP hydrolysis [7]. RecA also has domains responsible for monomer-monomer interaction, filament-filament interaction, and target protein binding. RecA crystallized as sixfold symmetric helical filament [57]. Additionally RecA upon binding to DNA was found to form filament like structures observed under TEM $[59,60]$.

Structural details of Dmcl alone and Dmc1-DNA complexes have been explored using TEM. Human Dmc1 was shown to form octameric ring-like structures and as stacked octameric rings upon binding to DNA $[43,44]$. It was observed that in the absence of ATP, human Dmcl formed stacked rings on DNA and in the presence of ATP, it formed helical filaments, which were active forms of recombinase-DNA complex [42]. It was also shown that $\mathrm{Ca}^{++}$promotes the pairing activity of yeast Dmcl by forming long helical filaments [61]. Crystal structure of human Dmc1 protein showed conserved outer and inner basic patches [62]. The inner basic patch was shown to bind ss and ds DNA; whereas outer basic patch binds only ssDNA. The $\mathrm{N}$-terminal 82 residues structure was found to be flexible in nature. The conserved Glu258 residue in hDmcl was probably responsible for octameric ring formation, which is not present in the Rad51 proteins. The hydrogen-bonding pattern guides the Rad51 to form hexameric rings.

Rad51 protein binds DNA to form helical filaments, which are similar to RecA [42, 63-66]. Yeast Rad51 protein formed helical filament structures only in presence of ATP and not in absence of ATP [67]. It has been shown that the filaments formed by yeast Rad51 protein were similar to RecA filaments [68]. Yeast Rad51 filament structure has been solved, and it was proposed that though the filament shows sixfold symmetry, the active form of filament has the threefold symmetry [69]. The ATP binding domain of protomers docks each other to form an active dimer. Three dimers together formed a helical filament with pseudo-sixfold symmetry. They also showed that His 352 is responsible for ssDNA binding and Tyr 112 is responsible for Rad52 interaction. A fusion protein Rad51-Brca2 complex has been crystallized, and it was shown that BRC repeat in Brca2 protein mimics the Rad51 oligomerization domain $[70,71]$. The resulted complex lacked the DNA binding activity and showed that BRC repeat is competing for the Rad51 oligomerization site. In conclusion, RecA, Dmc1, and Rad51 recombinases formed ring (hexameric/octameric) like structures in absence of DNA and upon binding to the DNA, they formed helical filaments.

\section{Recombination in Plants}

In plants homologous recombination occurs in meiosis and during somatic development. The meiotic HR is confined to coding regions; however, its frequency is not equal for all the genes, that is, different gene loci showed different recombination frequency $[72,73]$. The research in the area of homologous recombination in plants was driven by the desire targeting genes in transgenics. Several efforts were made to increase the frequency of homologous recombination in somatic cells in plants [74-77]. In most of these cases, regeneration of a functional marker gene for positive selection after recombination event was scored. These marker genes included antibiotic resistant genes like kanamycin, histochemical markers like $\beta$-glucuronidase, natural pigment genes like purple kernels in maize, and viral genomes like CaMV [78]. Using these markers, it was shown that the extrachromosomal somatic HR occurs at very high frequency $\left(10^{-1}\right)$. It has been also shown that the process depends on the topology of the molecules and length of the overlapping or homologous sequence. The process was shown to occur via single strand annealing (SSA) mediated recombination, in which one copy of the gene is lost and other is regenerated $[79,80]$. At the same time intrachromosomal somatic recombination was shown to occur at very low frequency in Arabidopsis and tobacco plants $\left(10^{-5}-10^{-6}\right)[81,82]$. However, different researchers have reported different frequencies. These differences may be due to the type of recombination substrate used, copy number of the gene, configuration of the gene, position of the transgene, and so forth [78]. Homologous recombination can lead to either crossover or gene conversion event depending on the mechanism of the resolution of the Holliday junction. In plant systems it has been reported that HR between the direct repeats yielded $67 \%$ gene conversions and 33\% crossovers [83]. However, between the inverted repeats, $90 \%$ gene conversion events are reported for tobacco plants [82]. Hence the mechanism of HR should allow the possibility of both gene conversion and crossover events. Double strand break repair model in yeast can explain these two events, and such mechanisms were also proposed for plants [78]. Apart from this conservative mechanism, other mechanisms like SSA have also been proposed. Both of these mechanisms may be operating simultaneously. 
Meanwhile, several genes related to homologous recombination process have been identified in plants by mutational analysis [84]. Xrs2 mutant in Arabidopsis thaliana plants showed hypersensitivity to DNA damaging agents like Xrays, MMC, and MMS with decreased somatic $H R$ and increased meiotic HR $[85,86]$. Mutations in Xrs9 and Xrs11 showed the same type of phenotypes as shown by Xrs2. Using reverse genetics approach, Rad9, Rad50, and Rad17 mutants have been characterized in Arabidopsis. These mutants show increased somatic HR, sensitivity to DNA damaging agents like MMS, MMC, UV radiation, bleomycine and defects in DSB repair mechanism [87-90]. In addition to this, like in yeast and mammals, RAD51 and DMC1 genes were also reported in plant system [91].

6.1. Plant DMC1. Based on the sequence information available for yeast Rad51 and Dmc1, using the degenerate oligonucleotide in RT-PCR method, Arabidopsis DMC1 gene was isolated [92-94]. Homologues of DMC1 have been also identified in Oryza sativa [95-98]. Using subtractive hybridization technique, a group of mRNAs was shown to accumulate during early stages of meiosis. Lim15 was detected during early prophase [99]. Sequence analysis showed Lim15 to be a putative protein product homologous to Dmc1 and Rad51. Immuno-localization studies on Rad51 and Lim15 on meiotic chromosomes showed that meiotic homologous recombination begins at leptotene and continues till zygotene stage [100]. In another study, it was shown that Dmcl formed foci and was involved in meiotic homology search. However, the species hybrid, where no homologous chromosome was found, corresponding decrease in synaptonemal complex was observed in Lily [101]. Although the expression of DMC1 was meiosis specific, some cultured cells also showed its expression. No increased expression of DMC1 was observed upon exposure to ionizing radiation $[93,94]$. Using the reverse genetic approach, T-DNA insertion in DMC1 gene under homozygous conditions showed reduced fertility and also resulted in random segregation of chromosomes in both male and female meiocytes [102]. When the functional wild type copy of the DMC1 was transferred to mutant plants, fertility was restored. This showed that $D M C 1$ is essential for meiosis.

$D M C 1$ from Japonica rice was isolated by RT-PCR method. It was observed that two homologues of OsDMC1 genes, namely, RiLIM15A and RiLIM15B were present in rice [95]. In the exonic regions, these two genes were highly homologous as compared to intronic regions. Southern hybridization experiments showed the expression of $D M C 1$ in meiotic tissue as well as in mature leaves. Additionally, transcript variants were reported where cDNA was lacking of exons 5, 8, 10, and 11. Simultaneously, using RT-PCR, rice $D M C 1$ was isolated and sequenced [97]. It was also shown that OsDMC1 expressed at high levels in reproductive tissues and at low levels in root tissues, but no expression was observed in mature leaves and seedlings. It was shown that the two rice DMC1 genes expressed differentially in haploid and diploid tissues [96]. Both the genes are expressed in pollen mother cells during meiosis, diploid tissue callus, and root tips. In addition to this, $D M C 1 B$ also expressed in hap- loid male gametes during pollen maturation, diploid zygotic embryos, and endosperm after pollination. This showed that $D M C 1 B$ alone or in conjunction with DMC1A meditated meiotic recombination. Recently using RNAi technology, it has been shown that OsDmc1 was required for homologous pairing in rice [103]. OsDMC1-RNAi lines grew normally during vegetative growth, but showed sterility. This was due to reduced levels of transcripts and protein. Cytological analysis showed that knockdown lines had defects in bivalent formation, resulted in random chromosome segregation and irregular spore formation. Fluorescent insitu hybridization showed that OsDMC1 was indeed required for homologous pairing. Biochemical analysis of OsDmc1 protein showed that the protein was functionally homologous to RecA type of recombinases $[104,105]$. cDNA corresponding to OsDMC1 from rice was expressed in E. coli. The purified recombinant OsDmclprotein was subjected to biochemical analysis. The binding affinity observed with circular ssDNA was similar to that with circular dsDNA and the binding was independent of presence of ATP. Even though the protein was binding to both the substrates equally well, only single-stranded DNA induced ATPase activity of the protein. OsDmc1-ssDNA complex also mediated renaturation of homologous complementary strands as well as D-loop formation activity. The D-loop formation was lowered by excess of OsDmc1 protein. Additionally, DNA renaturation and strand exchange activities of OsDmcl have been studied, using Fluorescence Resonance Energy Transfer (FRET). The level and the rate of renaturation was observed to be higher in presence of ATP. Using FRET assays, it was also shown that OsDmc1 protein catalyzed concentration dependent strand exchange reaction, where the activity was the fastest in the presence of ATP. All these results, put together, suggest that OsDmcl catalyses homologous renaturation as well as strand exchange events where ATP hydrolysis plays an important role. Recently Sakane et al. [106] studied the filament formation and strand exchange properties of OsDMC1A and OsDMC1B proteins. The purified recombinant OsDMC1A and OsDMC1B proteins upon on binding to DNA formed helical filamentous structures. In DNA binding assays, OsDMC1A protein was showed more affinity than that of OsDMC1B. Both the proteins promoted the strand exchange reaction up to several thousand base pairs in presence of the RPA. However in agreement with their DNA binding properties, OsDMC1A protein showed more strand exchange activity when compared with OsDMC1B. In future, these functional differences along with the difference in the sequences among the other plant DMC1 proteins, and structural information may provide the mechanistic aspects of the homologous recombination process in plant during meiosis.

6.2. Plant RAD51. Orthologues of Rad51 genes have been identified in several plants like Arabidopsis thaliana, Zea mays, Lycopersicon esculentum, and in moss Psyscomitrella patens. Arabidopsis genome has one copy of Rad51; whereas maize and Physcomitrella have two copies [93, 107-110]. After gamma irradiation it was shown that RAD51 transcription levels were increased in Arabidopsis [93]. It was also 
shown that Rad51 was dispensable for vegetative growth but was required during meiotic recombination [111]. RAD51 paralogs in Arabidopsis like AtRAD51B, AtRAD51C and AtXRCC2 and AtXRCC3 were shown to play a role in DNA repair and meiosis. AtRAD51C mutant showed defects in meiosis, sensitivity to mitomycin $\mathrm{C}$, but not to gammairradiation indicating that AtRAD51C may be involved in meiosis and mitosis [112-114]. AtRAD51B was shown to be involved in DNA repair process in somatic cells [115]. An interaction between AtRad51 and AtBrca2 was shown by Dray et al. [116]. In maize, $\operatorname{Rad} 51$ protein was shown to be involved in meiotic chromosome synapsis and segregation, supporting its involvement in meiotic homology search [117, 118]. In Japonica cultivar of rice two RAD51 genes have been reported ( $\mathrm{AB} 080262, \mathrm{AB} 080264$ ). These genes show 87\% homology at DNA level and 94\% homology at protein level.

The moss Physcomitralla patens showed higher frequency of homologous recombination as compared to higher plants [119]. Two copies of Rad51 genes have been detected in $P$. patens. The corresponding Rad51 proteins (PpaRad51.1 and PpaRad51.2) have been biochemicaly characterized by Ayora et al. [109]. Both the proteins shared 94\% identity and were shown to bind to single as well as double stranded DNA in the presence of $\mathrm{Mg}^{++}$and show strand annealing activity and strand exchange activity.

Analogues of Rad51 have also been detected in rice. The cDNA for Os Rad51 was cloned and overexpressed proteins were analyzed [120]. OsRad51 showed the classical hallmark biochemical properties of recombinase, which include ss and ds DNA binding in presence of $\mathrm{Mg}^{++}$forming helical filaments which mediated robust renaturation and strand exchange activities. These activities were homology sequence and ATP dependent. OsRad51 showed DNA dependent ATPase activity, which was stimulated by ssDNA. The strand assimilation property was measured using agarose gels and FRET assays. The renaturation activity was ATP and homology dependent in both the cases. OsRad51 also mediated strand exchange reaction between a duplex DNA and its homologous single stranded DNA, which was analyzed by FRET assays. OsRad51 showed protein concentration and time dependent strand exchange reaction, which was independent of ATP. The reaction products were stable even after deproteinization, and the reaction was homology dependent. Electron microscopy of OsRad51-DNA nucleoprotein complexes showed formation of DNA-protein filaments in presence and absence of ATP. Taken together, OsRad51 proteins showed the characteristic DNA binding, ATPase, renaturation, and strand exchange properties similar to classical recombinases. It will be interesting to study the functions of the recombinases in vivo using knockdown or knockout approaches to decipher the phenotypes of plants and also unravel the structure function relationship using high-resolution crystallographic data of Rad51 and Dmc1 proteins.

\section{Conclusions}

Though considerable progress has been made in knowledge on the prokaryotic and eukaryotic recombination process, it remains still an enigmatic process, especially in plants. Though explicit during meiosis and probably suppressed during mitosis, yet most vital for normal somatic development, for maintaining genomic stability during repair as well as for introduction of genetic variability during evolution. Since the amount of repetitive DNA is very high in plant species, plant genomes can be potentially recombinogenic. In spite of this, plants have less homologous recombination frequency as compared to yeast and mammals. Additionally differences in the frequency of homologous recombination between the different loci and varieties have been observed [78]. Additionally, there is need to increase frequency of homologous recombination for gene targeting in plants for using transgenic technology in plant breeding. Therefore understanding the structure-function relation, mechanism of action of DNA recombinases from plant system is important. Though the plant recombinases show apparent similarity in function to prokaryotic and eukaryotic recombinases, there are certain differences among the sequences. It will be interesting to investigate their structural and functional significance vis a vis the function of these recombinases as well as their exact role in determining the frequency of homologous recombination. It is relevant to point out that the exact mechanism of homology search at global genomic level is unclear. In E. coli, RecA protein performs the homology search and strand exchange. However, E. coli genome is small and it is easy to find the homologous sequence. But in case of yeast and higher eukaryotes, as genome size increases, probability of finding the homologous sequence decreases. There are two mechanisms proposed to explain the mechanism of homology search at genome level [121]. First one operates through ectopic recombination process, where homologous chromosomes and sequences align after DSB formation. This process is mediated by simple diffusion of molecules and takes more time. The second mechanism operates through prealigned homologous chromosome, where homologous chromosomes align prior to the DSB formation. This process is fast as compared to ectopic homology search, since molecules need not to diffuse. It was proposed that, in eukaryotes both the mechanisms operate, however the exact role of recombinases (Dmcl and Rad51) in this process is not known. We believe that recombinases in conjunction with other accessory proteins mediate the process of homology search at genome level. We conclude that further research in this area will improve our knowledge about the mechanism and regulation of the homologous recombination process in living systems, especially in plants, where this process has a long way to go.

\section{References}

[1] M. M. Cox, "Recombinational DNA repair of damaged repliction forks in Escherichia coli: questions," Annual Review of Genetics, vol. 35, pp. 53-82, 2001.

[2] L. S. Symington, "Role of RAD52 epistasis group genes in homologous recombination and double-strand break repair," Microbiology and Molecular Biology Reviews, vol. 66, no. 4, pp. 630-670, 2002. 
[3] A. J. Clark and A. D. Margulies, "Isolation and characterization of recombination-deficient mutants of E. coli K-12," Proceedings of the National Academy of Sciences of the United States of America, vol. 53, pp. 451-459, 1965.

[4] P. Howard-Flanders and L. Theriot, "Mutants of Escherichia coli $\mathrm{K}-12$ defective in DNA repair and in genetic recombination," Genetics, vol. 53, no. 6, pp. 1137-1150, 1966.

[5] A. Shinohara, H. Ogawa, and T. Ogawa, "Rad51 protein involved in repair and recombination in S. cerevisiae is a RecA-like protein," Cell, vol. 69, no. 3, pp. 457-470, 1992.

[6] F.-X. Barre, B. Soballe, B. Michel, M. Aroyo, M. Robertson, and D. Sherratt, "Circles: the replication-recombinationchromosome segregation connection," Proceedings of the National Academy of Sciences of the United States of America, vol. 98, no. 15, pp. 8189-8195, 2001.

[7] E. C. Kowalczykowski, D. A. Dixon, A. K. Eggleston, S. D. Lauder, and W. M. Rehrauer, "Biochemistry of homologous recombination in Escherichia coli," Microbiological Reviews, vol. 58, no. 3, pp. 401-465, 1994.

[8] G. M. Weinstock, K. McEntee, and I. R. Lehman, "ATPdependent renaturation of DNA catalyzed by the recA protein of Escherichia coli," Proceedings of the National Academy of Sciences of the United States of America, vol. 76, no. 1, pp. 126-130, 1979.

[9] F. R. Bryant and I. R. Lehman, "On the mechanism of renaturation of complementary DNA strands by the recA protein of Escherichia coli," Proceedings of the National Academy of Sciences of the United States of America, vol. 82, no. 2, pp. 297-301, 1985.

[10] S. S. Tsang, K. Muniyappa, E. Azhderian, et al., "Intermediates in homologous pairing promoted by recA protein: isolation and characterization of coli protein omega," Journal of Molecular Biology, vol. 55, pp. 523-533, 1985.

[11] F. R. Bryant, K. L. Menge, and T. T. Nguyen, "Kinetic modeling of the RecA protein promoted renaturation of complementary DNA strands," Biochemistry, vol. 28, no. 3, pp. 1062-1069, 1989.

[12] K. McEntee, G. M. Weinstock, and I. R. Lehman, "DNA and nucleoside triphosphate binding properties of recA protein from Escherichia coli," Progress in Nucleic Acid Research and Molecular Biology, vol. 26, pp. 265-279, 1981.

[13] J. P. Menetski and S. C. Kowalczykowski, "Interaction of recA protein with single-stranded DNA. Quantitative aspects of binding affinity modulation by nucleotide cofactors," Journal of Molecular Biology, vol. 181, no. 2, pp. 281-295, 1985.

[14] W. Rosselli and A. Stasiak, "Energetics of RecA-mediated recombination reactions: without ATP hydrolysis RecA can mediate polar strand exchange but is unable to recycle," Journal of Molecular Biology, vol. 216, no. 2, pp. 335-352, 1990.

[15] W. M. Rehrauer and S. C. Kowalczykowski, "Alteration of the nucleoside triphosphate (NTP) catalytic domain within Escherichia coli recA protein attenuates NTP hydrolysis but not joint molecule formation," The Journal of Biological Chemistry, vol. 268, no. 2, pp. 1292-1297, 1993.

[16] K. J. MacFarland, Q. Shan, R. B. Inman, and M. M. Cox, "RecA as motor protein," The Journal of Biological Chemistry, vol. 272, pp. 17675-17685, 1997.

[17] J.-Y. Masson and S. C. West, "The Rad51 and Dmc1 recombinases: a non-identical twin relationship," Trends in Biochemical Sciences, vol. 26, no. 2, pp. 131-136, 2001.

[18] A. Dudas and M. Chovanec, "DNA double-strand break repair by homologous recombination," Mutation Research, vol. 566, no. 2, pp. 131-167, 2004.
[19] G. Basile, M. Aker, and R. K. Mortimer, "Nucleotide sequence and transcriptional regulation of the yeast recombinational repair gene RAD51," Molecular and Cellular Biology, vol. 12, no. 7, pp. 3235-3246, 1992.

[20] P. Sung, "Catalysis of ATP-dependent homologous DNA pairing and strand exchange by yeast RAD51 protein," Science, vol. 265, no. 5176, pp. 1241-1243, 1994.

[21] D. K. Bishop, D. Park, L. Xu, and N. Kleckner, "DMC1: a meiosis-specific yeast homolog of E. coli recA required for recombination, synaptonemal complex formation, and cell cycle progression," Cell, vol. 69, no. 3, pp. 439-456, 1992.

[22] E. L. Hong, A. Shinohara, and D. K. Bishop, "Saccharomyces cerevisiae Dmcl protein promotes renaturation of singlestrand DNA (ssDNA) and assimilation of ssDNA into homologous super-coiled duplex DNA," The Journal of Biological Chemistry, vol. 276, no. 45, pp. 41906-41912, 2001.

[23] Y.-C. Chang, Y.-H. Lo, M.-H. Lee, et al., "Molecular visualization of the yeast Dmc1 protein ring and Dmc1-ssDNA nucleoprotein complex," Biochemistry, vol. 44, no. 16, pp. 6052-6058, 2005.

[24] T. Habu, T. Taki, A. West, Y. Nishimune, and T. Morita, "The mouse and human homologs of DMC 1, the yeast meiosisspecific homologous recombination gene, have a common unique form of exon-skipped transcript in meiosis," Nucleic Acids Research, vol. 24, no. 3, pp. 470-477, 1996.

[25] D. L. Pittman, J. Cobb, K. J. Schimenti, et al., "Meiotic prophase arrest with failure of chromosome synapsis in mice deficient for Dmc1, a germline-specific RecA homolog," Molecular Cell, vol. 1, no. 5, pp. 697-705, 1998.

[26] K. Yoshida, G. Kondoh, Y. Matsuda, T. Habu, Y. Nishimune, and T. Morita, "The mouse RecA-like gene Dmcl is required for homologous chromosome synapsis during meiosis," Molecular Cell, vol. 1, no. 5, pp. 707-718, 1998.

[27] A. Shinohara, H. Ogawa, Y. Matsuda, N. Ushio, K. Ikeo, and T. Ogawa, "Cloning of human, mouse and fission yeast recombination genes homologous to RAD51 and recA," Nature Genetics, vol. 4, no. 3, pp. 239-243, 1993.

[28] T. Morita, Y. Yoshimura, A. Yamamoto, et al., "A mouse homolog of the Escherichia coli recA and Saccharomyces cerevisiae RAD51 genes," Proceedings of the National Academy of Sciences of the United States of America, vol. 90, no. 14, pp. 6577-6580, 1993.

[29] Y. Yoshimura, T. Morita, A. Yamamoto, and A. Matsushiro, "Cloning and sequence of the human RecA-like gene cDNA," Nucleic Acids Research, vol. 21, no. 7, p. 1665, 1993.

[30] D.-S. Lim and P. Hasty, "A mutation in mouse rad51 results in an early embryonic lethal that is suppressed by a mutation in p53," Molecular and Cellular Biology, vol. 16, no. 12, pp. 7133-7143, 1996.

[31] S. Tashiro, N. Kotomura, A. Shinohara, K. Tanaka, K. Ueda, and N. Kamada, "S phase specific formation of the human Rad51 protein nuclear foci in lymphocytes," Oncogene, vol. 12, no. 10, pp. 2165-2170, 1996.

[32] J. Flygare, F. Benson, and D. Hellgren, "Expression of the human RAD51 gene during the cell cycle in primary human peripheral blood lymphocytes," Biochimica et Biophysica Acta, vol. 1312, no. 3, pp. 231-236, 1996.

[33] S. J. Xia, M. A. Shammas, and R. J. Shmookler Reis, "Elevated recombination in immortal human cells is mediated by HsRAD51 recombinase," Molecular and Cellular Biology, vol. 17, no. 12, pp. 7151-7158, 1997.

[34] E. A. Namsaraev and P. Berg, "Rad51 uses one mechanism to drive DNA strand exchange in both directions," The Journal of Biological Chemistry, vol. 275, no. 6, pp. 3970-3976, 2000. 
[35] E. A. Namsaraev and P. Berg, "Branch migration during Rad51-promoted strand exchange proceeds in either direction," Proceedings of the National Academy of Sciences of the United States of America, vol. 95, no. 18, pp. 10477-10481, 1998.

[36] P. Baumann, F. E. Benson, and S. C. West, "Human Rad51 protein promotes ATP-dependent homologous pairing and strand transfer reactions in vitro," Cell, vol. 87, no. 4, pp. 757766, 1996.

[37] R. C. Gupta, L. R. Bazemore, E. I. Golub, and C. M. Radding, "Activities of human recombination protein Rad51," Proceedings of the National Academy of Sciences of the United States of America, vol. 94, no. 2, pp. 463-468, 1997.

[38] J. K. Zutter and K. L. Knight, "The hRad51 and RecA proteins show significant differences in cooperative binding to singlestranded DNA," Journal of Molecular Biology, vol. 293, no. 4, pp. 769-780, 1999.

[39] A. V. Mazin, E. Zaitseva, P. Sung, and S. C. Kowalczykowski, "Tailed duplex DNA is the preferred substrate for Rad51 protein-mediated homologous pairing," The EMBO Journal, vol. 19 , no. 5, pp. 1148-1156, 2000.

[40] R. C. Gupta, E. Golub, B. Bi, and C. M. Radding, "The synaptic activity of $\mathrm{HsDmcl}$, a human recombination protein specific to meiosis," Proceedings of the National Academy of Sciences of the United States of America, vol. 98, no. 15, pp. 8433-8439, 2001.

[41] Z. Li, E. I. Golub, R. Gupta, and C. M. Radding, "Recombination activities of $\mathrm{HsDmcl}$ protein, the meiotic human homolog of RecA protein," Proceedings of the National Academy of Sciences of the United States of America, vol. 94, no. 21, pp. 11221-11226, 1997.

[42] M. G. Sehorn, S. Sigurdsson, W. Bussen, V. M. Unger, and P. Sung, "Human meiotic recombinase Dmcl promotes ATPdependent homologous DNA strand exchange," Nature, vol. 429, no. 6990, pp. 433-437, 2004.

[43] S. I. Passy, X. Yu, Z. Li, et al., "Human Dmcl protein binds DNA as an octameric ring," Proceedings of the National Academy of Sciences of the United States of America, vol. 96, no. 19, pp. 10684-10688, 1999.

[44] J.-Y. Masson, A. A. Davies, N. Hajibagheri, et al., "The meiosis-specific recombinase $\mathrm{hDmcl}$ forms ring structures and interacts with hRad51," The EMBO Journal, vol. 18, no. 22, pp. 6552-6560, 1999.

[45] M. C. Rice, S. T. Smith, F. Bullrich, P. Havre, and E. B. Kmiec, "Isolation of human and mouse genes based on homology to rec2, a recombinational repair gene from the fungus ustilago maydis," Proceedings of the National Academy of Sciences of the United States of America, vol. 94, no. 14, pp. 7417-7422, 1997.

[46] J. S. Albala, M. P. Thelen, C. Prange, et al., "Identification of a novel human RAD51 homolog, RAD51B," Genomics, vol. 46, no. 3, pp. 476-479, 1997.

[47] N. Liu, J. E. Lamerdin, R. S. Tebbs, et al., "XRCC2 and XRCC3, new human Rad51-family members, promote chromosome stability and protect against DNA cross-links and other damages," Molecular Cell, vol. 1, no. 6, pp. 783-793, 1998.

[48] M. Kawabata and K. Saeki, "Sequence analysis and expression of a novel mouse homolog of Escherichia coli recA gene," Biochimica et Biophysica Acta, vol. 1398, no. 3, pp. 353-358, 1998.

[49] M. K. Dosanjh, D. W. Collins, W. Fan, et al., "Isolation and characterization of RAD51C, a new human member of the RAD51 family of related genes," Nucleic Acids Research, vol. 26, no. 5, pp. 1179-1184, 1998.
[50] R. Cartwright, A. M. Dunn, P. J. Simpson, C. E. Tambini, and J. Thacker, "Isolation of novel human and mouse genes of the recA/RAD51 recombination-repair gene family," Nucleic Acids Research, vol. 26, no. 7, pp. 1653-1659, 1998.

[51] L. H. Thompson and D. Schild, "Recombinational DNA repair and human disease," Mutation Research, vol. 509, no. 1-2, pp. 49-78, 2002.

[52] Z. Shu, S. Smith, L. Wang, M. C. Rice, and E. B. Kmiec, "Disruption of muREC2/RAD51L1 in mice results in early embryonic lethality which can be partially rescued in a p532/2 background," Molecular and Cellular Biology, vol. 19, no. 12, pp. 8686-8693, 1999.

[53] D. L. Pittman and J. C. Schimenti, "Midgestation lethality in mice deficient for the RecA-related gene, Rad51d/Rad51l3," Genesis, vol. 26, no. 3, pp. 167-173, 2000.

[54] B. Deans, C. S. Griffin, M. Maconochie, and J. Thacker, "Xrcc2 is required for genetic stability, embryonic neurogenesis and viability in mice," The EMBO Journal, vol. 19, no. 24, pp. 6675-6685, 2000.

[55] S. Sigurdsson, K. Trujillo, B. Song, S. Stratton, and P. Sung, "Basis for Avid Homologous DNA strand exchange by human Rad51 and RPA," The Journal of Biological Chemistry, vol. 276, no. 12, pp. 8798-8806, 2001.

[56] Y.-C. Lio, A. V. Mazin, S. C. Kowalczykowski, and D. J. Chen, "Complex formation by the human Rad51B and Rad51C DNA repair proteins and their activities in vitro," The Journal of Biological Chemistry, vol. 278, no. 4, pp. 2469-2478, 2003.

[57] R. M. Story and T. A. Steitz, "Structure of the recA proteinADP complex,” Nature, vol. 355, no. 6358, pp. 374-376, 1992.

[58] R. M. Story, I. T. Weber, and T. A. Steitz, "The structure of the E. coli recA protein monomer and polymer," Nature, vol. 355, no. 6358, pp. 318-325, 1992.

[59] E. H. Egelman and A. Stasiak, "Structure of helical RecADNA complexes: complexes formed in the presence of ATPgamma-S or ATP," Journal of Molecular Biology, vol. 191, no. 4, pp. 677-697, 1986.

[60] E. H. Egelman and A. Stasiak, "Structure of helical RecADNA complexes-II: local conformational changes visualized in bundles of recA-ATP- $\gamma$-S filaments," Journal of Molecular Biology, vol. 200, no. 2, pp. 329-349, 1988.

[61] M.-H. Lee, Y.-C. Chang, E. L. Hong, et al., "Calcium ion promotes yeast Dmcl activity via formation of long and fine helical filaments with single-stranded DNA," The Journal of Biological Chemistry, vol. 280, no. 49, pp. 40980-40984, 2005.

[62] T. Kinebuchi, W. Kagawa, R. Enomoto, et al., "Structural basis for octameric ring formation and DNA interaction of the human homologous-pairing protein Dmc1," Molecular Cell, vol. 14, no. 3, pp. 363-374, 2004.

[63] F. E. Benson, A. Stasiak, and S. C. West, "Purification and characterization of the human Rad51 protein, an analogue of E. coli RecA," The EMBO Journal, vol. 13, no. 23, pp. 57645771, 1994.

[64] P. Baumann and S. C. West, "Role of the human RAD51 protein in homologous recombination and double-strandedbreak repair," Trends in Biochemical Sciences, vol. 23, no. 7, pp. 247-251, 1998.

[65] P. Chi, S. Van-Komen, M. G. Sehorn, S. Sigurdsson, and P. Sung, "Roles of ATP binding and ATP hydrolysis in human Rad51 recombinase function," DNA Repair, vol. 5, no. 3, pp. 381-391, 2006.

[66] V. E. Galkin, F. Esashi, X. Yu, S. Yang, S. C. West, and E. H. Egelman, "BRCA2 BRC motifs bind RAD51-DNA filaments," Proceedings of the National Academy of Sciences of the United States of America, vol. 102, no. 24, pp. 8537-8542, 2005. 
[67] P. Sung and D. L. Robberson, "DNA strand exchange mediated by a RAD51-ssDNA nucleoprotein filament with polarity opposite to that of RecA," Cell, vol. 82, no. 3, pp. 453-461, 1995.

[68] T. Ogawa, X. Yu, A. Shinohara, and E. H. Egelman, "Similarity of the yeast RAD51 filament to the bacterial RecA filament," Science, vol. 259, no. 5103, pp. 1896-1899, 1993.

[69] A. B. Conway, T. W. Lynch, Y. Zhang, et al., "Crystal structure of a Rad51 filament," Nature Structural and Molecular Biology, vol. 11, no. 8, pp. 791-796, 2004.

[70] A. A. Davies, J.-Y. Masson, M. J. McIlwraith, et al., "Role of BRCA2 in control of the RAD51 recombination and DNA repair protein," Molecular Cell, vol. 7, no. 2, pp. 273-282, 2001.

[71] L. Pellegrini, D. S. Yu, T. Lo, et al., "Insights into DNA recombination from the structure of a RAD51-BRCA2 complex," Nature, vol. 420, no. 6913, pp. 287-293, 2002.

[72] X. Xu, A. P. Hsia, L. Zhang, B. J. Nikolau, and P. S. Schnable, "Meiotic recombination break points resolve at high rates at the 5 ' end of a maize coding sequence," The Plant Cell, vol. 7, pp. 2151-2161, 1995.

[73] G. I. Patterson, K. M. Kubo, T. Shroyer, and V. L. Chandler, "Sequences required for paramutation of the maize $b$ gene map to a region containing the promoter and upstream sequences," Genetics, vol. 140, no. 4, pp. 1389-1406, 1995.

[74] A. Peterhans, H. Schlupmann, C. Basse, and J. Paszkowski, "Intrachromosomal recombination in plants," The EMBO Journal, vol. 9, no. 11, pp. 3437-3445, 1990.

[75] S. Gal, B. Pisan, T. Hohn, N. Grimsley, and B. Hohn, "Genomic homologous recombination in planta," The EMBO Journal, vol. 10, no. 6, pp. 1571-1578, 1991.

[76] P. Swoboda, S. Gal, B. Hohn, and H. Puchta, "Intrachromosomal homologous recombination in whole plants," The EMBO Journal, vol. 13, no. 2, pp. 484-489, 1994.

[77] H. Puchta, P. Swoboda, S. Gal, M. Blot, and B. Hohn, "Somatic intrachromosomal homologous recombination events in populations of plant siblings," Plant Molecular Biology, vol. 28, no. 2, pp. 281-292, 1995.

[78] H. Puchta and B. Hohn, "From centiMorgans to base pairs: homologous recombination in plants," Trends in Plant Science, vol. 1, no. 10, pp. 340-348, 1996.

[79] R. Offringa, M. J. A. de Groot, H. J. Haagsman, M. P. Does, P. J. M. Van den Elzen, and P. J. J. Hooykaas, "Extrachromosomal homologous recombination and gene targeting in plant cells after Agrobacterium mediated transformation," The EMBO Journal, vol. 9, no. 10, pp. 3077-3084, 1990.

[80] B. Tinland, B. Hohn, and H. Puchta, "Agrobacterium tumefaciens transfers single-stranded transferred DNA (TDNA) into the plant cell nucleus," Proceedings of the National Academy of Sciences of the United States of America, vol. 91, no. 17 , pp. 8000-8004, 1994.

[81] P. Athma and T. Peterson, "Ac induces homologous recombination at the maize P locus," Genetics, vol. 128, no. 1, pp. 163-173, 1991.

[82] J. Tovar and C. Lichtenstein, "Somatic and meiotic chromosomal recombination between inverted duplications in transgenic tobacco plants," The Plant Cell, vol. 4, no. 3, pp. 319-332, 1992.

[83] F. F. Assaad and E. R. Signer, "Somatic and germinal recombination of a direct repeat in Arabidopsis," Genetics, vol. 132, no. 2, pp. 553-566, 1992.

[84] D. Schuermann, J. Molinier, O. Fritsch, and B. Hohn, "The dual nature of homologous recombination in plants," Trends in Genetics, vol. 21, no. 3, pp. 172-181, 2005.
[85] J. E. Masson, P. J. King, and J. Paszkowski, "Mutants of Arabidopsis thaliana hypersensitive to DNA-damaging treatments," Genetics, vol. 146, no. 1, pp. 401-407, 1997.

[86] J. E. Masson and J. Paszkowski, "Arabidopsis thaliana mutants altered in homologous recombination," Proceedings of the National Academy of Sciences of the United States of America, vol. 94, no. 21, pp. 11731-11735, 1997.

[87] M. E. Gallego, M. Jeanneau, F. Granier, et al., "Disruption of the Arabidopsis RAD50 gene leads to plant sterility and MMS sensitivity," The Plant Journal, vol. 25, no. 1, pp. 31-41, 2001.

[88] H. Gherbi, M. E. Gallego, N. Jalut, J. M. Lucht, B. Hohn, and C. I. White, "Homologous recombination in planta is stimulated in the absence of Rad50," EMBO Reports, vol. 2, no. 4, pp. 287-291, 2001.

[89] M. E. Gallego and C. I. White, "RAD50 function is essential for telomere maintenance in Arabidopsis," Proceedings of the National Academy of Sciences of the United States of America, vol. 98, no. 4, pp. 1711-1716, 2001.

[90] F. Heitzeberg, I.-P. Chen, F. Hartung, N. Orel, K. J. Angelis, and H. Puchta, "The Rad17 homologue of Arabidopsis is involved in the regulation of DNA damage repair and homologous recombination," The Plant Journal, vol. 38, no. 6, pp. 954-968, 2004.

[91] J.-Y. Bleuyard, M. E. Gallego, and C. I. White, "Recent advances in understanding of the DNA double-strand break repair machinery of plants," DNA Repair, vol. 5, no. 1, pp. 1-12, 2006.

[92] S. Sato, Y. Hotta, and S. Tabata, "Structural analysis of a recA-like gene in the genome of Arabidopsis thaliana," DNA Research, vol. 2, no. 2, pp. 89-93, 1995.

[93] M.-P. Doutriaux, F. Couteau, C. Bergounioux, and C. White, "Isolation and characterisation of the RAD51 and DMC1 homologs from Arabidopsis thaliana," Molecular and General Genetics, vol. 257, no. 3, pp. 283-291, 1998.

[94] V. I. Klimyuk and J. D. G. Jones, "AtDMC1, the Arabidopsis homologue of the yeast DMC1 gene: characterization, transposon-induced allelic variation and meiosis-associated expression," The Plant Journal, vol. 11, no. 1, pp. 1-14, 1997.

[95] J. Shimazu, C. Matsukura, M. Senda, et al., "Characterization of a DMC1 homologue, RiLIM15, in meiotic panicles, mitotic cultured cells and mature leaves of rice (Oryza sativa L.)," Theoretical and Applied Genetics, vol. 102, no. 8, pp. 1159$1163,2001$.

[96] A. Kathiresan, G. S. Khush, and J. Bennett, "Two rice DMC1 genes are differentially expressed during meiosis and during haploid and diploid mitosis," Sexual Plant Reproduction, vol. 14, no. 5, pp. 257-267, 2002.

[97] Z.-J. Ding, T. Wang, K. Chong, and S. Bai, "Isolation and characterization of OsDMC1, the rice homologue of the yeast DMC1 gene essential for meiosis," Sexual Plant Reproduction, vol. 13, no. 5, pp. 285-288, 2000.

[98] S. S. Metkar, J. K. Sainis, and S. K. Mahajan, "Cloning and characterization of the DMC1 genes in Oryza sativa," Current Science, vol. 87, no. 3, pp. 353-357, 2004.

[99] T. Kobayashi, E. Kobayashi, S. Sato, et al., "Characterization of cDNAs induced in meiotic prophase in lily microsporocytes," DNA Research, vol. 1, no. 1, pp. 15-26, 1994.

[100] M. Terasawa, A. Shinohara, Y. Hotta, H. Ogawa, and T. Ogawa, "Localization of RecA-like recombination proteins on chromosomes of the lily at various meiotic stages," Genes and Development, vol. 9, no. 8, pp. 925-934, 1995.

[101] S. George, P. Behl, R. DeGuzman, et al., "Dmc1 fluorescent foci in prophase I nuclei of diploid, triploid and hybrid lilies," Chromosoma, vol. 111, no. 2, pp. 96-105, 2002. 
[102] F. Couteau, F. Belzile, C. Horlow, O. Grandjean, D. Vezon, and M.-P. Doutriaux, "Random chromosome segregation without meiotic arrest in both male and female meiocytes of a dmc1 mutant of Arabidopsis," The Plant Cell, vol. 11, no. 9, pp. 1623-1634, 1999.

[103] Z. Y. Deng and T. Wang, "OsDMC1 is required for homologous pairing in Oryza sativa," Plant Molecular Biology, vol. 65, no. 1-2, pp. 31-42, 2007.

[104] C. R. Kant, B. J. Rao, and J. K. Sainis, "DNA binding and pairing activity of OsDmc1, a recombinase from rice," Plant Molecular Biology, vol. 57, no. 1, pp. 1-11, 2005.

[105] C. Rajanikant, M. Kumbhakar, H. Pal, B. J. Rao, and J. K. Sainis, "DNA strand exchange activity of rice recombinase OsDmc1 monitored by fluorescence resonance energy transfer and the role of ATP hydrolysis," FEBS Journal, vol. 273, no. 7, pp. 1497-1506, 2006.

[106] I. Sakane, C. Kamataki, Y. Takizawa, et al., "Filament formation and robust strand exchange activities of the rice DMC1A and DMC1B proteins," Nucleic Acids Research, vol. 36, no. 13, pp. 4266-4276, 2008.

[107] N. Y. Stassen, J. M. Logsdon Jr., G. J. Vora, H. H. Offenberg, J. D. Palmer, and M. E. Zolan, "Isolation and characterization of rad51 orthologs from Coprinus cinereus and Lycopersicon esculentum, and phylogenetic analysis of eukaryotic recA homologs," Current Genetics, vol. 31, no. 2, pp. 144-157, 1997.

[108] A. E. Franklin, J. McElver, I. Sunjevaric, R. Rothstein, B. Bowen, and W. Z. Cande, "Three-dimensional microscopy of the Rad51 recombination protein during meiotic prophase," The Plant Cell, vol. 11, no. 5, pp. 809-824, 1999.

[109] S. Ayora, J. I. Piruat, R. Luna, et al., "Characterization of two highly similar Rad51 homologs of Physcomitrella patens," Journal of Molecular Biology, vol. 316, no. 1, pp. 35-49, 2002.

[110] U. Markmann-Mulisch, M. Z. Hadi, K. Koepchen, et al., "The organization of Physcomitrella patens RAD51 genes is unique among eukaryotic organisms," Proceedings of the National Academy of Sciences of the United States of America, vol. 99, no. 5, pp. 2959-2964, 2002.

[111] W. Li, C. Chen, U. Markmann-Mulisch, et al., "The Arabidopsis AtRAD51 gene is dispensable for vegetative development but required for meiosis," Proceedings of the National Academy of Sciences of the United States of America, vol. 101, no. 29, pp. 10596-10601, 2004.

[112] K. Abe, K. Osakabe, S. Nakayama, et al., "Arabidopsis RAD51C gene is important for homologous recombination in meiosis and mitosis," Plant Physiology, vol. 139, no. 2, pp. 896-908, 2005.

[113] J.-Y. Bleuyard, M. E. Gallego, F. Savigny, and C. I. White, "Differing requirements for the Arabidopsis Rad51 paralogs in meiosis and DNA repair," The Plant Journal, vol. 41, no. 4, pp. 533-545, 2005.

[114] W. Li, X. Yang, Z. Lin, et al., "The AtRAD51C gene is required for normal meiotic chromosome synapsis and doublestranded break repair in Arabidopsis," Plant Physiology, vol. 138, no. 2, pp. 965-976, 2005.

[115] K. Osakabe, K. Abe, H. Yamanouchi, et al., "Arabidopsis Rad51B is important for double-strand DNA breaks repair in somatic cells," Plant Molecular Biology, vol. 57, no. 6, pp. 819-833, 2005.

[116] E. Dray, N. Siaud, E. Dubois, and M.-P. Doutriaux, "Interaction between Arabidopsis Brca2 and its partners Rad51, Dmc1, and Dss1," Plant Physiology, vol. 140, no. 3, pp. 10591069, 2006.
[117] A. E. Franklin, I. N. Golubovskaya, H. W. Bass, and W. Z. Cande, "Improper chromosome synapsis is associated with elongated RAD51 structures in the maize desynaptic2 mutant," Chromosoma, vol. 112, no. 1, pp. 17-25, 2003.

[118] W. P. Pawlowski, I. N. Golubovskaya, and W. Z. Cande, "Altered nuclear distribution of recombination protein RAD51 in maize mutants suggests the involvement of RAD51 in meiotic homology recognition," The Plant Cell, vol. 15, no. 8, pp. 1807-1816, 2003.

[119] D. G. Schaefer and J.-P. Zryd, "Efficient gene targeting in the moss Physcomitrella patens," The Plant Journal, vol. 11, no. 6, pp. 1195-1206, 1997.

[120] C. Rajanikant, M. Melzer, B. J. Rao, and J. K. Sainis, "Homologous recombination properties of OsRad51, a recombinase from rice," Plant Molecular Biology, vol. 68, no. 4-5, pp. 479491, 2008.

[121] A. Barzel and M. Kupiec, "Finding a match: how do homologous sequences get together for recombination?" Nature Reviews Genetics, vol. 9, no. 1, pp. 27-37, 2008. 

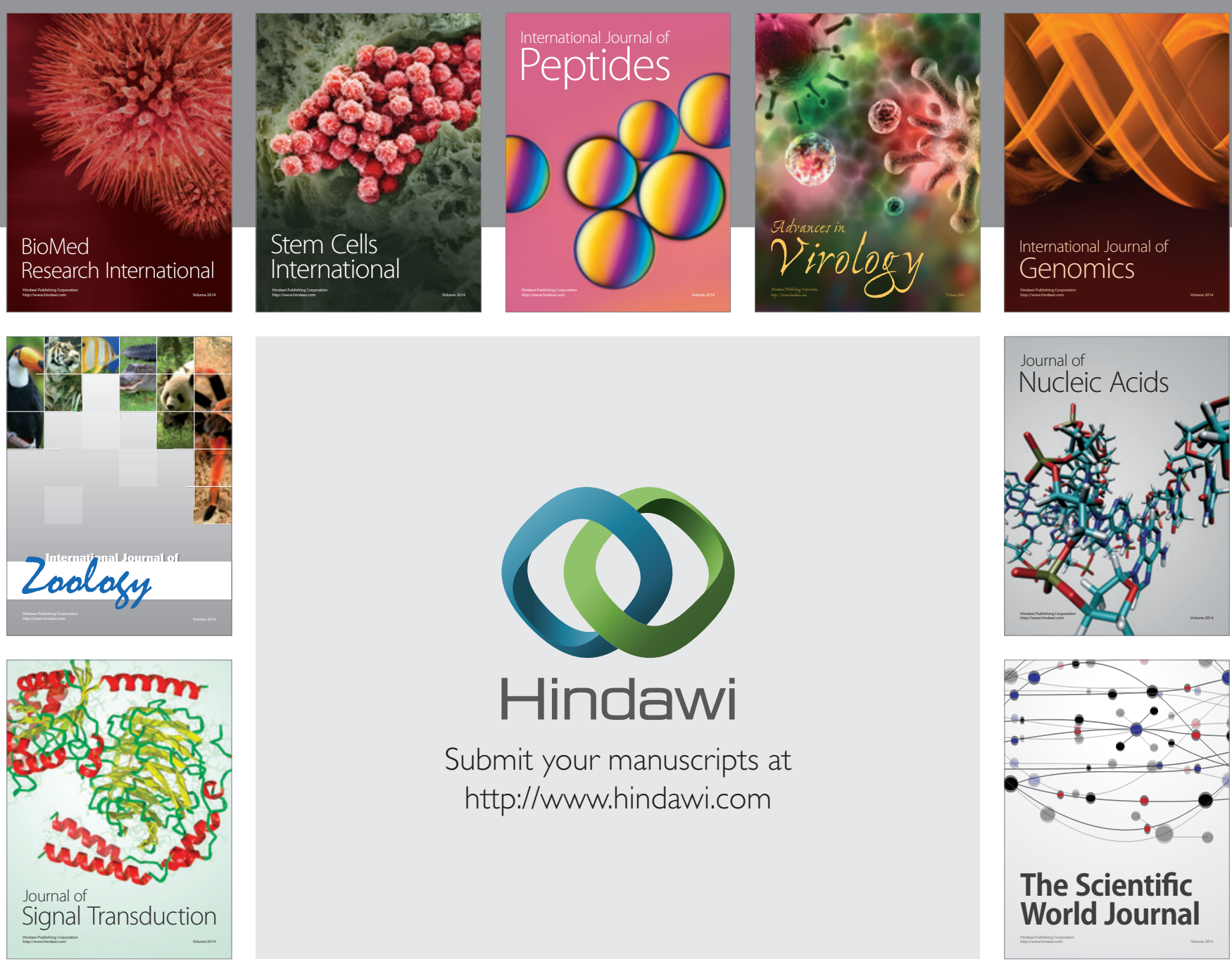

Submit your manuscripts at

http://www.hindawi.com
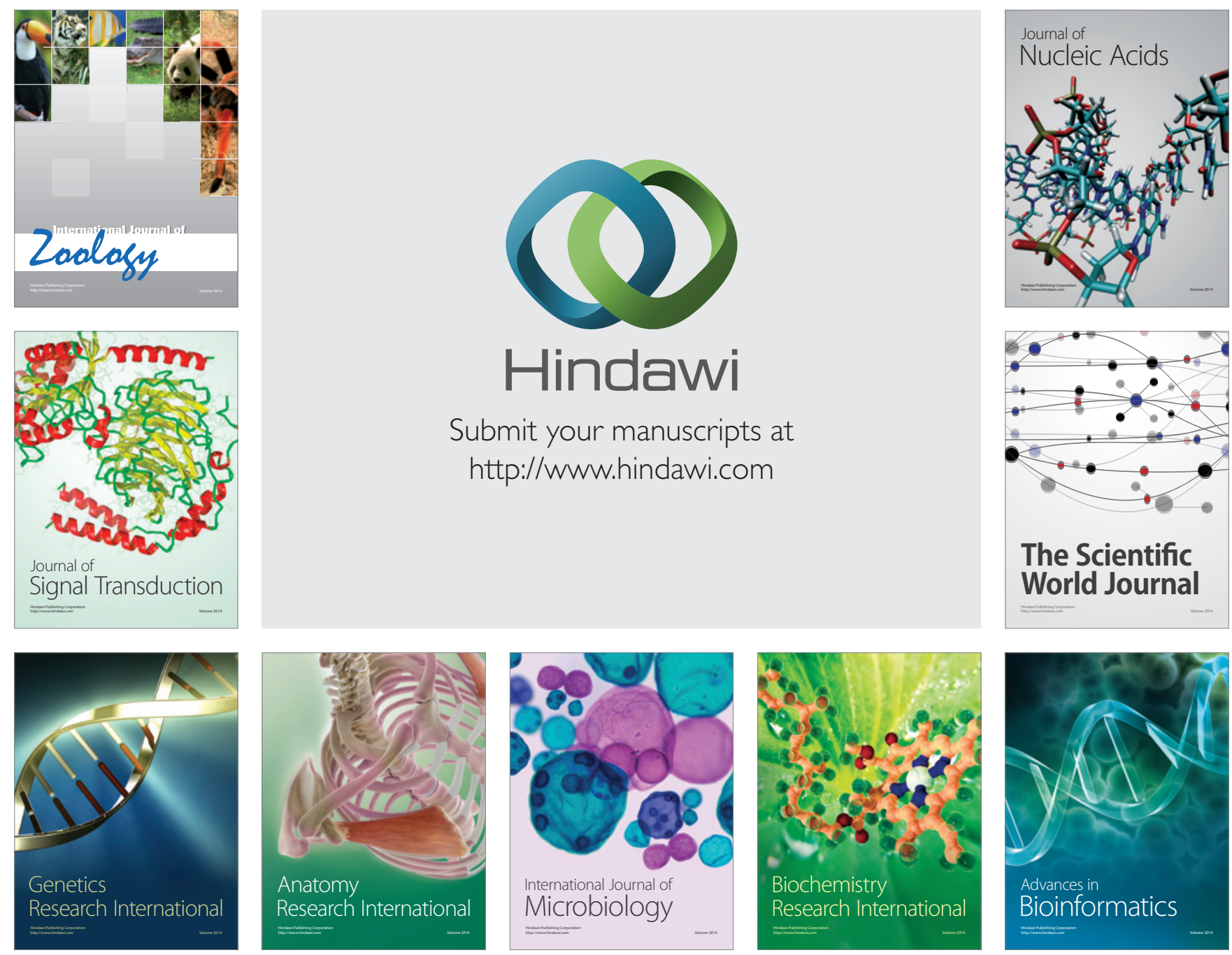

The Scientific World Journal
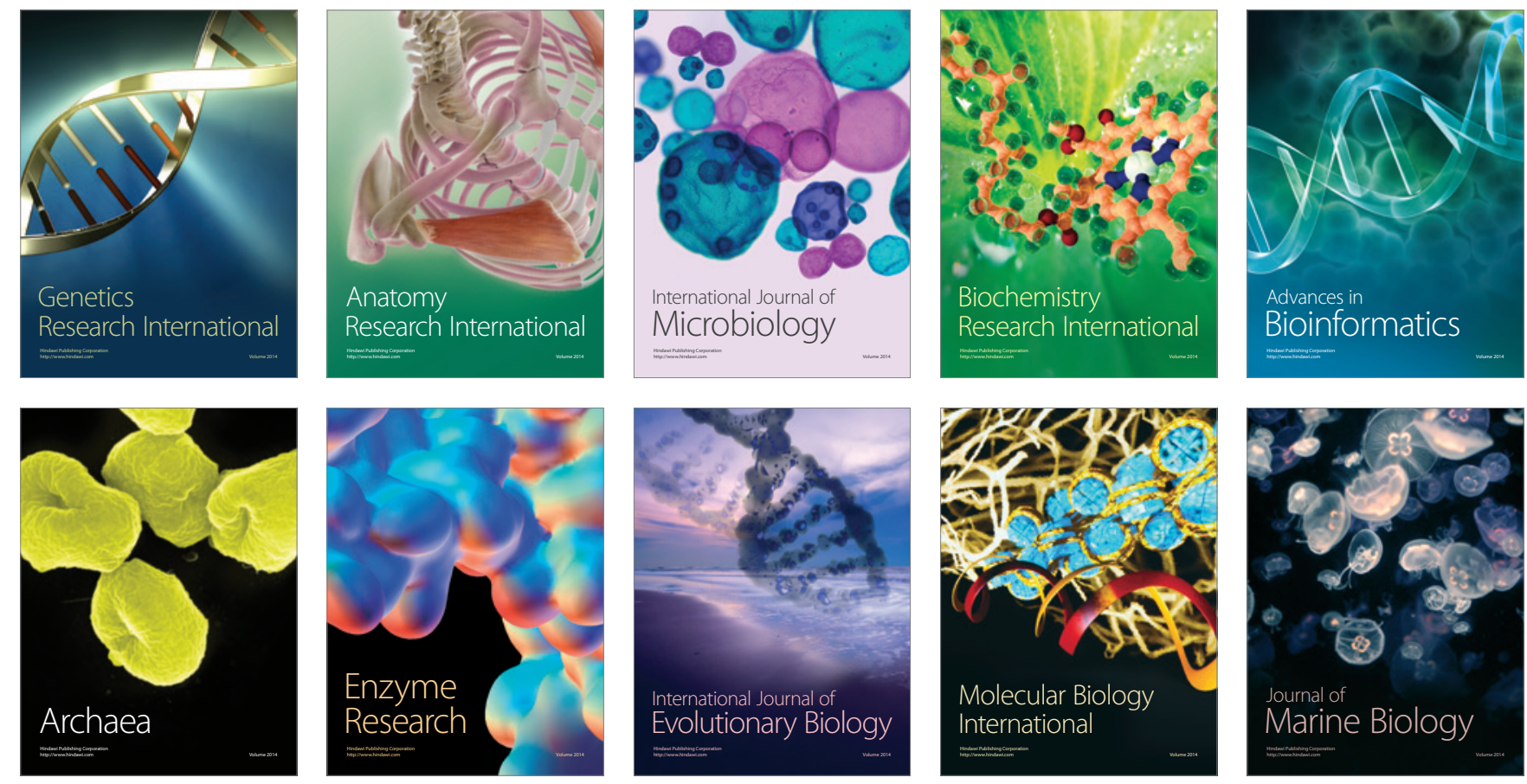